\title{
ELECTRICAL MODEL IN HELIUM DIELECTRIC BARRIIER DISCHARGE (DBD)*
}

\author{
Heba S. Gabr**, Mohamed A. El Shaer
}

Physics and Mathematics Eng. Dept., Faculty of Engineering, Zagazig University, Egypt

\begin{abstract}
:
An electric model created by Simulink/Matlab is used to describe the main characteristics of a plasma dielectric barrier discharge (DBD) at atmospheric pressure under a flow of helium gas. The model is based on a voltage controlled current source and a variable gap capacitor. The applied voltage, the corresponding current, gap voltage and dielectric voltage are the main characteristic investigated in the present work. The applied sinusoidal voltage to the electrodes is generated by an induction coil transformer at $5.7 \mathrm{kHz}$. Experimental measurements are made to extract some DBD parameters and validate the model results.
\end{abstract}

\section{KEY WORDS:}

\section{ELECTRIQUES MODELE EN HELIUM DÉCHARGE DIÉLECTRIQUE BARRIIER (DBD)}

\section{RÉSUMÉ :}

Un modèle électrique créé par Simulink / Matlab est utilisé pour décrire les principales caractéristiques d'une décharge de plasma à barrière diélectrique (DBD) à la pression atmosphérique sous un flux d'hélium gazeux. Le modèle est basé sur une source de tension commandée en courant et un condensateur de décalage variable. La tension appliquée, le courant correspondant, tension d'espace et de tension diélectrique sont la principale caractéristique étudiée dans le présent travail. La tension sinusoïdale appliquée aux électrodes est produit par une bobine d'induction du transformateur à 5,7 kHz. Les mesures expérimentales sont faites pour extraire certains paramètres DBD et de valider les résultats du modèle.

\section{MOTS CLÉS:}

\footnotetext{
* Received: 11/10/2012, Accepted: 14/11/2012, Ref. No. 126, (Original Paper)

** Contact author (engheba_gabr@yahoo.com, +20 1119893490)
} 
ELECTRICAL MODEL IN HELIUM DIELECTRIC BARRIIER DISCHARGE (DBD)

Gabr, El Shaer

\section{INTRODUCTION}

Atmospheric pressure plasma generated by dielectric barrier discharges (DBD) have been extensively studied and widely used for various industrial applications, [1]. The DBD is used to produce non-thermal plasma in reactor type geometry. DBD consists of low-temperature plasma discharge working at atmospheric or near atmospheric pressure and generated between two parallel metallic electrodes, one of them or both are covered by dielectric medium and separated by an air gap of some millimetres. The plasma can be generated in the gap by the gas excitation due to the application of alternating current (ac) voltage with frequency in the range of few $\mathrm{kHz}$ to several $\mathrm{MHz}$. The dielectric barrier can be made from glass, quartz, ceramics, or polymer materials of low dielectric loss and high breakdown strength, [2].

The applications of DBD range from thin layer deposition and sputtering [3], surface modifications of materials such as plastics and polypropylene [4], and biomedical applications, $[5,6]$.

In order to use such type of discharge in industrial scale, the parameters of the discharge in various conditions should be identified. Many efforts have been done to describe the discharge appearance of DBD. Hasina khatun, [7] reports the filamentary behaviour of a coaxial dielectric barrier discharge lamp by varying applied voltage, pressure and frequency. L.Oksuz, [8] realizes that dielectric barrier discharge can change from streamer to glow discharge by altering the frequency. R. Kozakov, [9] investigates the properties of DBD in different gas mixture. Z. Fang, [10] founds that the voltage range for sustaining homogenous stable discharge is wider for thin barrier thickness or small gap distance.

Proper equivalent electrical model has been wide spread used for studying DBD characteristics including external operating parameters, reactor configuration and gas properties, [11-17]. Plasma discharge has been represented by two series capacitors connected to variable resistor [14] or connected to two Zener diodes in parallel [11] or to voltage controlled current source $[12,16]$.

In this paper, discharge characteristics of an atmospheric pressure (DBD) in helium driven by high voltage ignition coil of frequency $5.7 \mathrm{KHz}$ simulation by electrical model are developed. . The adopted electrical model follow the model in [16], modelling our experimental conditions. Many discharge characteristics of interest will be investigated especially the total DBD voltage and corresponding current at certain value of discharge gap distance as well as gas voltage, dielectric voltage. Those characteristics are taken for several gap distances. This should give a complete picture of DBD's supporting their applications in plasma aided manufacturing and industrial purposes. 


\section{DBD ELECTRICAL MODEL}

One of the simplest ways to estimate the electrical plasma parameters of the DBD cell is the "electrical modelling". Electrical modelling is mainly done by representing each part of plasma DBD system by its equivalent element to perform electrical circuit model used to understand plasma discharge behaviour and simulate current and voltage waveforms. The computer program mostly used to implement electrical characterization of DBD is Simulink by Matlab [13, 15-17], and it has been used in this work. The plasma electrical circuit model uses the measured voltage and current to determine the system resistance in real time, and to validate the simulated results of plasma discharge.

\subsection{DBD Equivalent Circuit:}

Electrical circuit of helium DBD in open air is shown in Figure1. The DBD cell consists of two plate-capacitors connected in series as in Fig. 1a. A dielectric glass of dielectric barrier capacitance $C_{d}$ covers the powered electrode, whereas the helium gap is described by capacitance $C_{g}$.

The equations used to calculate the values of the capacitances $C_{d}$ and $C_{g}$, not including plasma discharge, are:

$$
\begin{aligned}
& d=\frac{\epsilon_{0} \in_{r 1} A}{\left(L_{d}\right)} \\
& g=\frac{\epsilon_{0} \epsilon_{r 2} A}{\left(L_{g}\right)}
\end{aligned}
$$

Where $\quad \epsilon_{0}=8.854 * 10^{-12} \mathrm{~F} / \mathrm{m} \quad$ vacuum permittivity, $\epsilon_{r 1}=4.7$ and $\epsilon_{r 2}=1.000065$ are Pyrex glass and helium relative permittivity respectively. $L_{d}$, dielectric thickness of $2 \mathrm{~mm}$ and, $L_{g}$, is the gap thickness which can be adjusted from $1 \mathrm{~mm}$ to $27 \mathrm{~mm}$ and $A$ is the area of electrode plate .

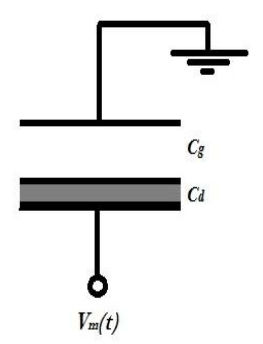

(a)

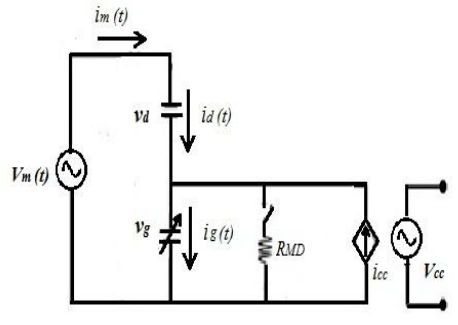

(b)
Fig. 1: Equivalent electrical Circuit of DBD Cell

Before the sinusoidal applied voltage $V_{m}(t)$ reaches certain point called break down voltage $V_{b d}$ (even in the positive or negative half cycle of the sinusoidal waveform $\left.V_{m}(t)\right)$, no ionization occurs and the DBD cell equivalent circuit is represented as pure capacitors. Since $d$ and $C_{g}$ are in serial connection, the equivalent capacitance is calculated as:

$$
e q=\frac{C_{d} C_{g}}{C_{d}+C_{g}}
$$

The total current $i_{m}(t)$ passed through the DBD reactor is equal to dielectric current $i_{d}(t)$ and gap current $i_{g}(t)$. In Fig. 1, after applied voltage exceeds the breakdown value, the phenomenon of micro discharges will be represented for a particular time interval, and the variation of ionization level leads to non linear 


\section{ELECTRICAL MODEL IN HELIUM DIELECTRIC BARRIIER DISCHARGE (DBD)}

Gabr, El Shaer

change of gap capacitance $C_{g}$. Therefore gap capacitance $C_{g}$ has been presented by variable capacitor $C_{g}(t)$.

The non linear change of gap capacitance $C_{g}$ is due to the relative permittivity of gas, which decreases when the ionization ignite and return to its natural value when the ionization extinct, [18]. For helium working gas, the gap capacitance reduces to $70 \%$ of its original value as stated in [16], so it has been decided in electrical model that gap capacitance during discharge equal to $0.7 C_{g}$.

In addition to $C_{g(t)}, R_{M D}$ and $i_{c c s}(t)$ are introduced to take into account the micro discharges phenomena. Where $R_{M D}$ represent the resistance of the micro discharge which is parallel connected to $C_{g(t)}$, and $i_{c c s}(t)$ represent the micro discharge current which passes through $R_{M D}$ and is produced by a currentcoltage controlled source (CCS). The value of $i_{c c s}(t)$ depends on the applied voltage $V_{m}(t)$, as it is will be mentioned in the following section.

\subsection{DBD Governing Equations:}

According to Kirchhoff's Law, the voltage and current applied to the DBD can be calculated from Fig. 1-b, using the following equation :

$$
\begin{aligned}
& V_{m}(t)=v_{d}(t)+v_{g}(t) \\
& i_{m}(t)=i_{d}(t)=d \frac{d v_{d}(t)}{d t} \\
& i_{g}(t)=C_{g} \frac{d v_{g}(t)}{d t}
\end{aligned}
$$

The total current $i_{m}(t)$ is consisting of conduction current $i_{c c s}(t)$ and displacement current through the plasma capacitance $i_{g}(t)$ [18].

$$
i_{m}(t)=i_{c c s}(t)+i_{g}(t)
$$

By differentiating equation (4),

$$
\frac{d V_{m}(t)}{d t}=\frac{d v_{g}(t)}{d t}+\frac{d v_{d}(t)}{d t}
$$

Substituting (5), (6) and (7) in (8) and rearranging,

$$
i_{c c s}(t)=\left(1+\frac{g}{d}\right) i_{m}(t)-C_{g} \frac{d V_{m}(t)}{d t}
$$

So, it can be seen from equation (9) that $i_{c c s}(t)$ is controlled by the applied voltage $V_{m}(t)$.

By integrating (5) and rearranging, we obtain the dielectric voltage $v_{d}(t)$,

$$
v_{d}(t)=\frac{1}{d} \int_{0}^{t} i_{m}(\tau) d \tau+v_{d}(0)
$$

Substitute (10) in (4) and get the gap voltage $v_{g}(t)$,

$$
v_{g}(t)=V_{m}(t)-\frac{1}{d} \int_{0}^{t} i_{m}(\tau) d \tau-v_{d}(0)
$$

Where $v_{d}(0)$ is the initial voltage on $C_{d}$ and corresponds to the memory voltage due to charge accumulation on the dielectric surface in the previous half cycle. The memory voltage $v_{d}(0)$ depends on the peak of the applied voltage $V_{m}$ as calculated in [19], where $\mathrm{T}$ is the period of one cycle.

$$
\begin{aligned}
v_{d}(0)=-\frac{1}{2 C_{d}} \int_{0}^{\frac{T}{2}} i_{m}(\tau) d \tau \\
=-\frac{g}{g+d} V_{m} \\
-\frac{\int_{0}^{\frac{T}{2}} i_{c c s}(\tau) d \tau}{2(g+d)}
\end{aligned}
$$


When the applied voltage polarity is reversed.

The $V_{m}(t)$ is added to a memory voltage $v_{d}(0)$ and the gap voltage is maintained, which is sufficiently high for the gap to break down at low values of applied voltage. Therefore the sustaining voltage for the DBD is obviously lower than the ignition voltage required for the first time [16]. So, for the first breakdown, the value of memory voltage $v_{d}(0)$ is zero, but for the next half cycle it will be non zero.

\subsection{Discharge Duration:}

The applied voltage $V_{m}(t)$ is described by a sinusoidal waveform equation:

$V_{m}(t)=V_{m} \sin (\omega t)$

Where $V_{m}$ is the amplitude of the applied voltage and $\omega$ is the operating angular frequency in $\mathrm{rad} / \mathrm{sec}$.

Once the amplitude of applied voltage exceeds breakdown voltage $V_{b d}$, the helium gap particles are ionized, plasma is formed, discharge current is generated and its intensity increased abruptly due to the dynamics of the gas conductivity. It is assumed that the duration from $t_{1}$ to $t_{2}$ is the time of micro discharge in positive half cycle and from $t_{3}$ to $t_{4}$ in negative half cycle, where $t_{1}$ and $t_{3}$ are called ignition moment which occurred at breakdown voltage ${ }_{-}^{+} V_{b d}$,respectively. $\quad t_{2}$ and $t_{4}$ one called extinction moment which occurred at maximum amplitude of applied voltage ${ }_{-} V_{m}$, respectively [16].

So, we can say:

$V_{m}\left(t_{1}\right)=V_{b d}$

$V_{m}\left(t_{3}\right)=-V_{b d}$

$V_{m}\left(t_{2}\right)=V_{m}$

$V_{m}\left(t_{4}\right)=-V_{m}$

After rearranging those equations we get:

$$
\begin{aligned}
t_{1} & =\frac{1}{\omega}\left(\sin ^{-1}\left(\frac{V_{b d}}{V_{m}}\right)\right)_{\text {radian }} \\
t_{3} & =\frac{1}{\omega}\left(\sin ^{-1}\left(\frac{-V_{b d}}{V_{m}}\right)\right)_{\text {radian }} \\
t_{2} & =\frac{T}{4} \\
t_{4} & =\frac{3 T}{4}
\end{aligned}
$$

The breakdown voltage $V_{b d}$, is the value of the applied voltage when ignition of the discharges occurs and its obtain experimentally, section 3 .

\subsection{Electrical Model Mechanism}

An electrical model shown in Fig. 2, implements the equivalent circuit DBD cell of Fig.1 by MATLAB /Simulink. To describe electrical model in detail, firstly we should know that the important part in this model is the representation of micro discharge mechanism. Where, parameters including ignition and extinction of micro discharge are taken into account. 


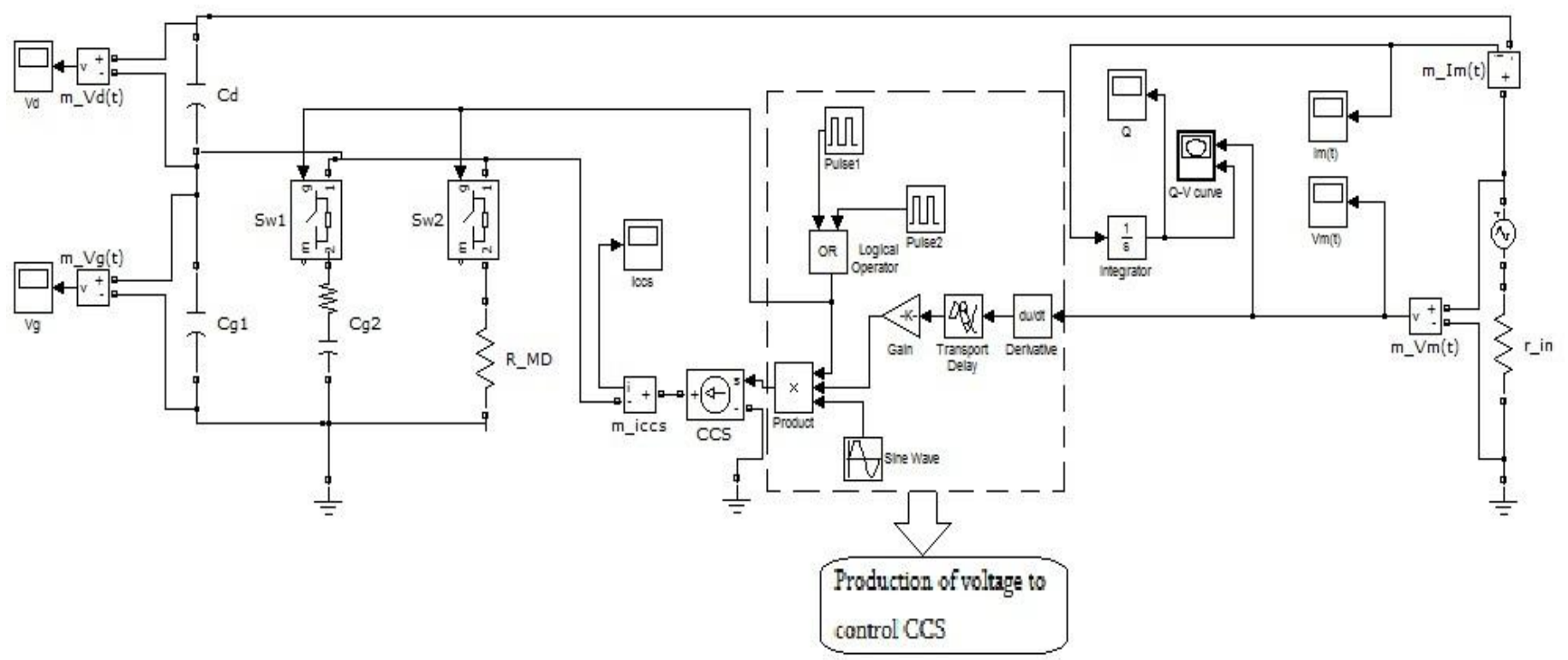

Fig. 2: Electrical model simulation of DBD using MATLAB/SIMULINK

Applied voltage $V_{m}(t)$ in the model consists of a sinusoidal wave and resistance $r_{i n}$, which represents the internal resistance of ignition coils Fig. 2. Internal resitance $r_{\text {in }}$ has been set by $6.67 \mathrm{KOhm}$ as in [20], where sinusoidal wave $6 \mathrm{KV}$ peak and frequency $5.7 \mathrm{KHz}$.

Pulse1 and Pulse 2 are two important blocks, which operating when applied voltage exceeds the breakdown voltage and used to describe the duration of discharge. Where its output is periodical pulse of unity amplitude, and it duration in positive half cycle from $t_{1}$ to $t_{2}$ for Pulse1, and in negative half cycle from $t_{3}$ to $t_{4}$ for Pulse2. Those values of times can be obtained from equations (18-21). "Or Block", work as on-off control of discharge. It was used to collect the outputs of Pulse1 and Pulse 2 and get Boolean output of ' 1 ' during discharge is on, and ' 0 ' during discharge is off.
Variable gap capacitor and Micro Discharge resistance are presented by $C_{g 1}, C_{g 2}$ and $R_{M D}$, respectively. The non linear change of gap capacitor is obtained by dividing $g$ into ${ }_{g 1}=0.7 C_{g}$ and $C_{g 2}=0.3 C_{g}$, where ${ }_{g 1}$, is the capacitor during discharge and $g_{1}+g_{2}$ are pure value of gap capacitance after discharge extinction as mention in section 2.1. To express this mechanism we use two ideal switches $S_{w 1}$ and $S_{w 2}$ which are controlled by trigger signal of "Or Block". Those switches are programmed as, when $S_{w 1}$ is open, $S_{w 2}$ is closed then $R_{M D}$ connect in parallel with $C_{g 1}$. This case presents the micro discharge region of DBD cell and those elements depend on applied gas composition. Within extinction and next ignition of discharge, $S_{w 1}$ is closed and $S_{w 2}$ is opened then $R_{M D}$ disconnected and $g_{2}$ is in parallel 
with $g_{1}$ which equivalent topology is the sum of two capacitance and equal to $C_{g}$.

The value of $R_{M D}$ has been evaluated as follows, [15].

$$
\frac{V_{g_{b r}}}{I_{m}}
$$

Where $V_{g_{b r}}$ is the gap breakdown voltage and $I_{m}$ is the peak of total discharge current $i_{m}(t)$. $V_{g_{b r}}$ can be calculated from paschen equation, which depends on gap distance $L_{g}$ and gas pressure $\mathrm{P},[18]$.

$$
V_{g_{b r}}=\frac{D^{2} P L_{g}}{\left[\ln \left(C p L_{g}\right)-\ln \left(\ln \left(1+\frac{1}{\gamma}\right)\right)\right]^{2}}
$$

$\gamma$ is the secondary electron emission coefficient which depends on the cathode material. C and D are constants which depend on gas type asfollows. $C=44$ Torr $\mathrm{cm}^{-1}$,

$D=14 \mathrm{~V} /$ Torr $^{\frac{1}{2}} \mathrm{Cm}^{\frac{1}{2}}$, and $\gamma=0.01$. These values give good agreement between experimental gap breakdown voltages in helium DBD and calculated value using equation (23) for a range of frequencies in order of few $\mathrm{KHz}$, [21]. The discharge occurs in atmospheric pressure $\mathrm{P}=760$ Torr. In consequence, according to value of gap distance $L_{g}$, gap breakdown voltage can be detected.

$V_{\text {CCS }}$ segment represents the dotted region shown in Fig. 2. This segment illustrates the construction of voltage which used to control current controlled source, CCS, as to form the conduction current $i_{c c s}(t)$ during discharge region. From equation (9) $i_{c c s}(t)$ depends on derivative of the applied voltage $\frac{d V_{m}(t)}{d t}$, so we express it by derivative block which its input is the applied voltage $V_{m}(t)$. The output of derivative block is function of sin wave shifted by $\pi / 2$ and amplitude of $\omega V_{m}$. So to get unity output with zero phase shifts we use gain block and phase delay block to do this job, respectively. Multi peaks appearing in the total current $i_{m}(t)$ during micro discharge are similar to sinusoidal waveform Fig. 4. Therefore, "Sin Block" is very useful to express these ripples. Number of peaks which is produced in one half cycle of current depends on discharge duration. Our goal is to get DBD discharge characteristics which will be simulated by this model. Those characteristics are total current $i_{m}(t)$, applied voltage $V_{m}(t)$, gas gap voltage $v_{g}(t)$ and dielectric voltage $v_{d}(t)$,

\section{EXPERIMENTAL SETUP AND MEASUREMENTS}

Fig. 3, shows the schematic view of the discharge chamber of the DBD reactor. The discharge occurs on a cross shaped Pyrex tube of $11 \mathrm{~cm}$ inner diameter and $30 \mathrm{~cm}$ arm length which can be evacuated by a rotary vacuum pump. The electrodes are two circular shaped Stainless-steel plates facing one another of diameter $9 \mathrm{~cm}$. The inter-electrode distance can be varied from zero up to few centimetres.

Helium gas of purity $99.999 \%$ can be injected through the upper electrode connected to ground. The lower electrode is connected to the 
power supply and covered by a circular glass plate of thickness $2 \mathrm{~mm}$.

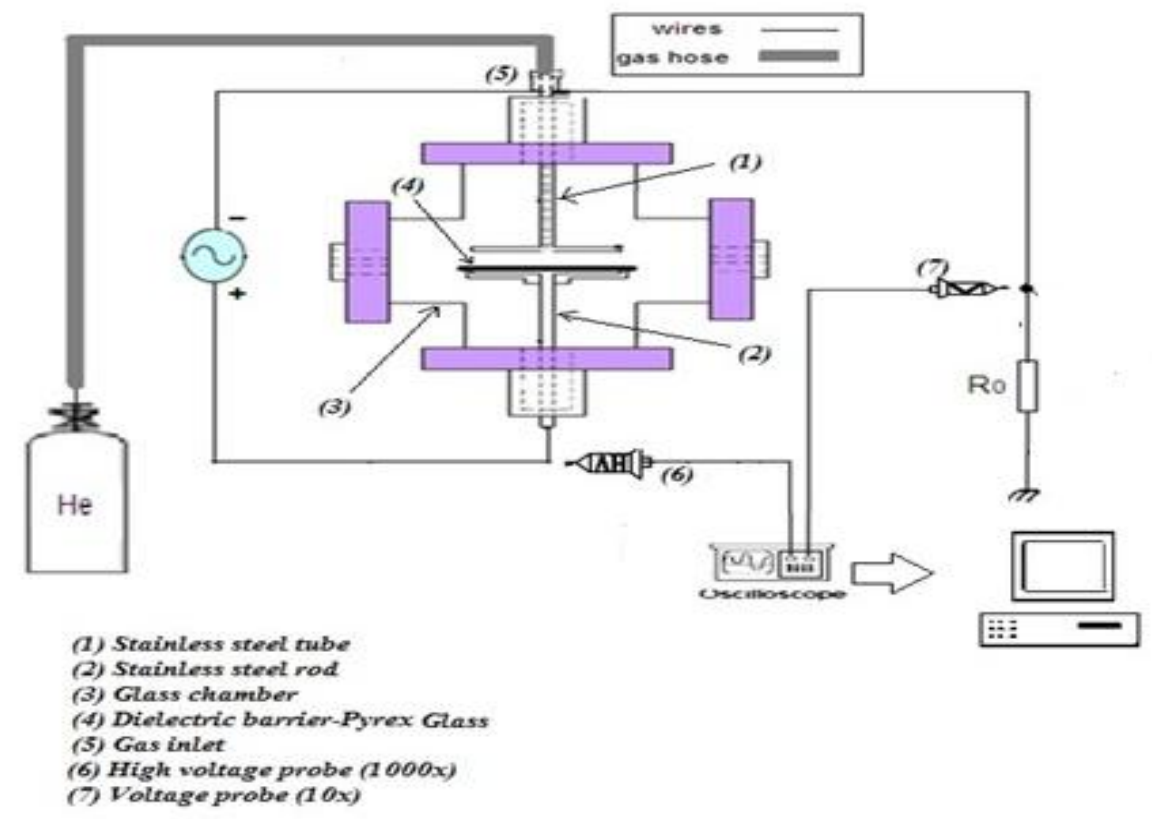

Fig. 3: Experimental setup

The discharge is created by the application of low frequency sinusoidal high voltage signal between the two electrodes. The high voltage up to $6 \mathrm{kV}$ peak with frequency $5.7 \mathrm{kHz}$ is delivered by an ignition transformer coil equipped with a driver circuit.

During operation, a primary vacuum pumping is carried out in the chamber until a pressure of 10 mbar is reached then pure helium is introduced raising the working pressure up to 1000 mbar.

The electrical measurements is made using a high voltage probe (Tektronix P6015A, bandwidth: $75 \mathrm{MHz}$ and ratio: 1000) to measure the voltage across the DBD cell. An external resistance $\mathrm{R}_{0}(50 \mathrm{ohm})$ connected to ground are used to measure the total current on the DBD cell. This value of resistance is chosen in a way to produce negligible effect on the impedance of micro discharges. The applied voltage and the total current waveforms are visualized by a GDS-840S digital oscilloscope. The oscilloscope is interfaced with a personal computer recording the obtained pattern waveforms. Fig. 4 shows voltage-current wave forms for a discharge with different gap distances. The object of getting those waveforms is to determine the value of peak value of discharge current $i_{m}(t)$, and the applied break down voltage $\mathrm{V}_{\mathrm{bd}}$. Applied breakdown voltage is marked by the value of total DBD voltage before the drop in voltage wave form in half cycle, where the corresponding values for 5,9 and $17 \mathrm{~mm}$ gap distance are 960, 1120 and $1800 \mathrm{~V}$, respectively, and corresponding discharge currents are $10.8,12$ and $17.2 \mathrm{~mA}$ for the same gaps. 

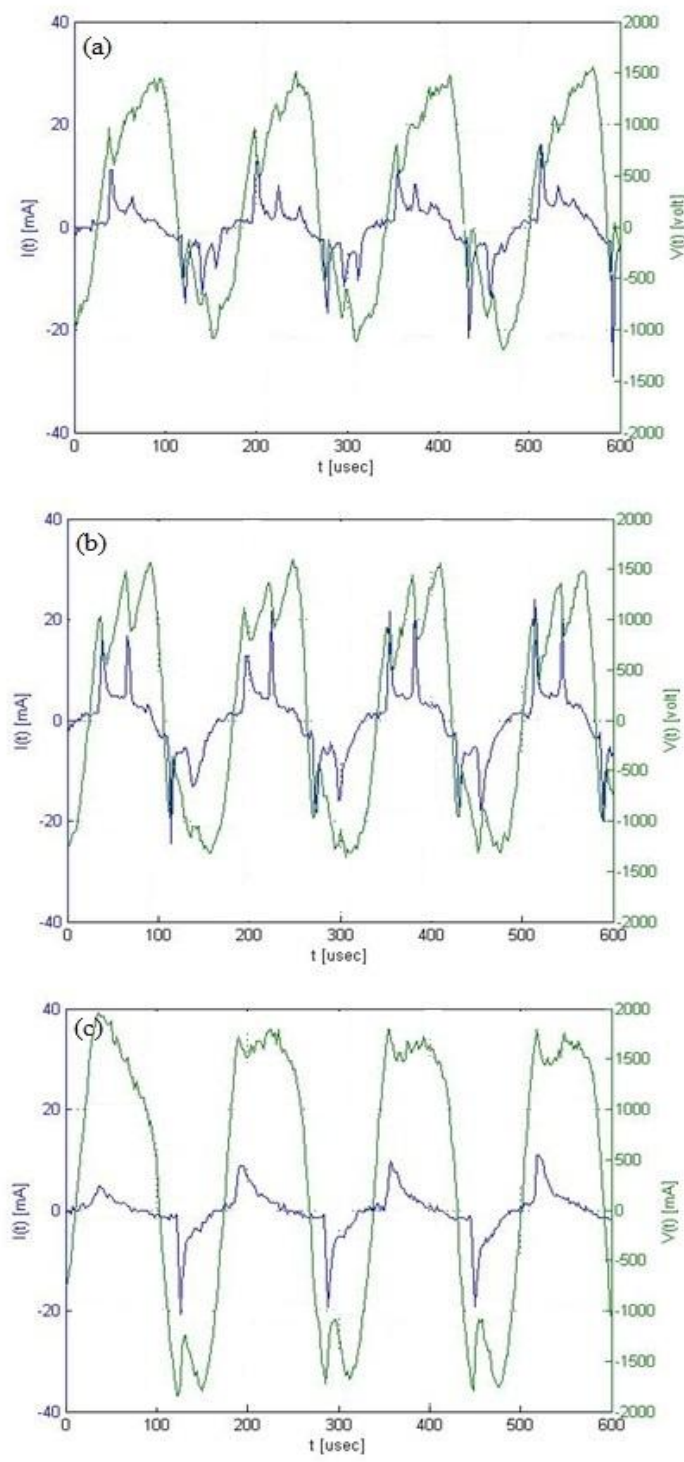

Fig. 4: Experimental results of applied voltage and total current waveform at gap distance $\mathrm{L}_{\mathrm{g}}$ (a) $5 \mathrm{~mm}$, (b) $9 \mathrm{~mm}$, and (c) $17 \mathrm{~mm}$

\section{RESULTS AND DISCUSSION:}

Calculated model parameter inputs, $\left(C_{d}, C_{g 1}, C_{g 2}, R_{M D}, t_{1}\right.$ and $\left.t_{2}\right)$ at each value of gas gap distance are given in Table1.This is done by using the equations $(1,2,18,19$, 22,23). Scope block has been created in model to get our results of electrical characteristics. From table 1 we see that $R_{M D}$ is increased with increasing gap distance and the capacitance of plasma gas is decreased with the increasing of gap distance. These variations lead the peak value of applied voltage $V_{m}(t)$ to increase with increasing gap distance as in Fig.4. Error! Reference source not found. represents simulation model results of total current $i_{m}(t)$ and applied voltage $V_{m}(t)$ according to the variation of gap distance at atmospheric pressure under flowing helium for a frequency of 5.7 KHz. The importance is mainly on overall performance of simulation in terms of amplitude and nature of discharge peak. It has been detected that numbers of peaks increase with decreasing gap distance. In fact, this due to the discharge duration, that was indicated in last column of table1.

The gas gap voltage $v_{g}(t)$ and dielectric voltage $v_{d}(t)$ has been simulated in electrical model by connecting the scope block across the gap capacitance $C_{g}$ and dielectric capacitance $C_{d}$, respectively. Error! Reference source not found. shows the characteristic of different voltages for DBD cell in simulated model. The drop in the gap voltage marks the ignition condition, where the discharge occurs when applied voltage reaches the breakdown voltage and results in significant electron production. After that, the produced electrons move towards momentary anode driven by gap voltage. It can be noticed that the dielectric voltage $v_{d}(t)$ is 
ELECTRICAL MODEL IN HELIUM DIELECTRIC BARRIIER DISCHARGE (DBD)

Gabr, El Shaer

decreasing with increasing gap distance [22].

This is due to the increase in the break down

voltage, which cause to raise the gap voltage $v_{g}(t)$, and according to equation (4) the $v_{d}(t)$ reduced. It is clear evident that the gap voltage attains positive value prior to applied voltage which confirms the effect of memory voltage.

Table 1: Model Parameter Inputs

\begin{tabular}{|c|c|c|c|c|c|c|c|}
\hline $\begin{array}{l}L_{g} \\
(\mathrm{~mm})\end{array}$ & ${ }_{d}(\mathrm{~F})$ & $g 1(\mathrm{~F})$ & $g_{2}(\mathrm{~F})$ & $\begin{array}{l}R_{M D} \\
(\mathrm{~K} \Omega)\end{array}$ & $t_{1}(\mathrm{sec})$ & $t_{3}(\mathrm{sec})$ & $\begin{array}{l}\left(t_{2}-t_{1}\right) f \\
* 100 \%\end{array}$ \\
\hline 5 & & 0.00787 & 0.00337 & 99.02 & $2.038 * 10^{-5}$ & $1.081 * 10^{-4}$ & $13.39 \%$ \\
\hline 9 & 0.14153 & 0.004377 & 0.00187 & 101 & $2.165^{*} 10^{-5}$ & $1.097 * 10^{-4}$ & $12.7 \%$ \\
\hline 17 & & 0.002127 & 0.0011 & 297.375 & $3.57 * 10^{-5}$ & $1.234 * 10^{-4}$ & $4.7 \%$ \\
\hline
\end{tabular}
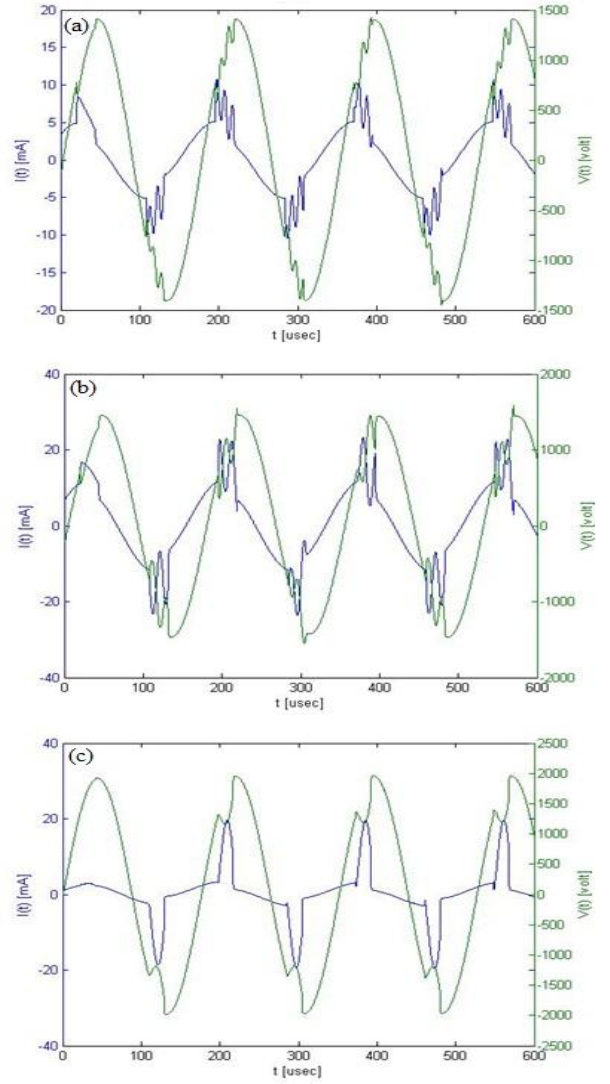

Fig. 5: Model result of total current $I(t)$ and DBD voltage $V_{m}$ (t) (a) $5 \mathrm{~mm}$ gap, (b) $9 \mathrm{~mm}$, (c) $17 \mathrm{~mm}$ gap distance
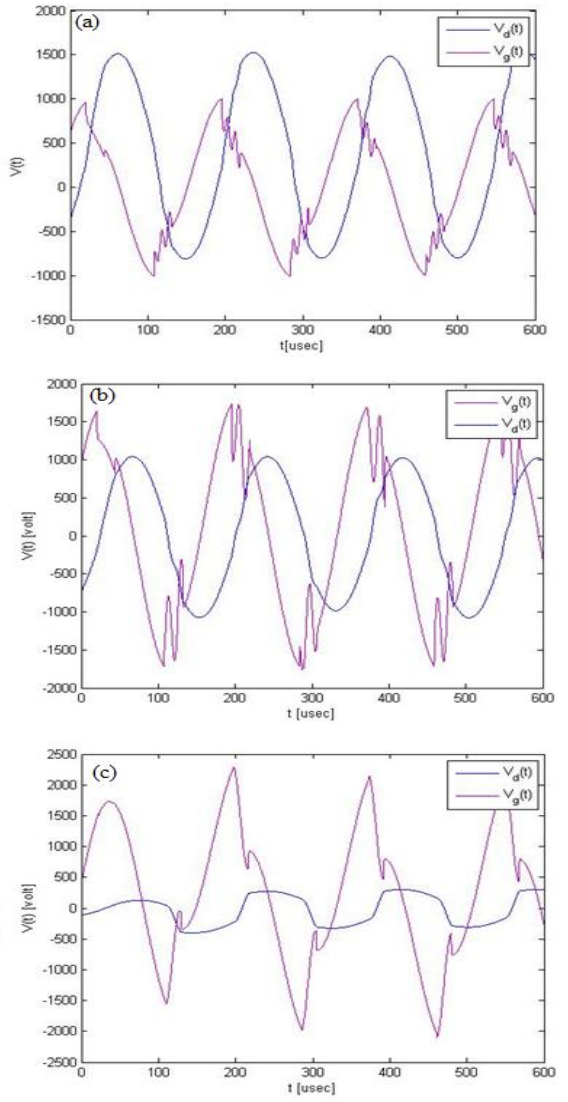

Fig. 6: gap voltage $V_{g}(t)$ and dielectric voltage $V_{d}(t)$ at gap distance $17 \mathrm{~mm}$ at (a) $5 \mathrm{~mm}$ gap, (b) $9 \mathrm{~mm},(\mathrm{c}) 17 \mathrm{~mm}$ gap distance 


\section{CONCLUSION}

An electrical circuit has been proposed to analyze internal electrical parameters for DBD at atmospheric pressure. The equations based on the equivalent electrical circuit have been established. The discharge characteristics of current, total voltage dielectric voltage and gas gap voltage have been simulated in different gap distance.

A computer model for electrical circuit has been implemented in the MATLAB Simulink. The operation of each block in MATLAB electrical model has been illustrated in details. The experiment has been performed using helium DBD, as the electrical circuit model uses the measured voltage and current to determine the system resistance in real time, and to validate the simulated results of plasma discharge Multi pulses result from repeated breakdown events in the discharge and its number decreased with increasing the gap distance.

\section{REFERENCES}

[1] Kogelschatz U, Eliason B and Egli W. Dielectric barrier discharges: principle and applications. J Phys IV France 1997; 7(C4): 4766.

[2] Chirokov A, Gutsol A, and Fridman A. Atmospheric pressure plasma of dielectric barrier discharges. Pure Appl Chem 2005; 77(2): 487-95.

[3] Wagner H.-E, Brandenburg R, Kozlov KV and Behnke JF. The barrier discharge: basic properties and applications to surface treatment. Vacuum 2003; 71 (3): 417-36.
[4] Bogaertsa A, Neyts E, Gijbels Rt. Review of Gas discharge plasmas and their applications. Spectrochimica Acta Part B 2002; 57:609-58

[5] Polk C. Biological applications of large electric fields: Some history and fundamentals. IEEE Trans Plasma Sci.2000; 28(1): 4-16.

[6] Goree J, Liu B, Drake D, and Stoffels E. Killing of S. mutans bacteria using a plasma needle at atmospheric pressure. IEEE Trans Plasma Sci.2006; 34(4): 1317-24.

[7] Khatun H, Mishra A, Kumar M and Barhai PK. Study of filamentary behaviour in coaxial dielectric barrier discharge lamp. Indian J Pure and Appl Phys 2008; 46: 889-92.

[8] Oksuz L, Korkmaz S, helhel S, Atilmis Y and Ellingboe AR. Dielectric barrier discharge Transition from Streamer to Glow. In:Proc.XXVIIth ICPIG, Eindhoven, the Netherlands; 2005.

[9] Kozakov R, Sonnenfeld A and Behnke JF. Investigation of the dielectric barrier discharge properties in different gas mixtures. Czech $\mathbf{J}$ Phys 2000; 50(S3).

[10] Fang Z, Qiu Y, Zhang C and Kuffel E. Factors influencing existence of the homogeneous dielectric barrier discharge in air at atmospheric pressure. J Phys D: Appl Phys 2007; 40(5): 1401-7.

[11] Naude N, Cambreonne JP, Gherardi N and Massines F. Electrical model and analysis of the transition from an atmospheric pressure Townsend discharge to a filamentary discharge. J Phys D:Appl Phys 2005;38(4): 530-38.

[12] Chen ZY. Pspice simulation of one atmospheric uniform glow discharge plasma (OAUGDP) reactor systems, IEEE Trans Plasma Sci 2003; 31 (4): 511-20.

[13] Flores-Fuentes A, Pena-Eguiluz R, LopezCallejas R and Valencia-Alvarado R. "Electrical Model of an atmospheric Pressure Dielectric Barrier Discharge Cell. IEEE Trans Plasma Sci 2009. ;37(1):128-134. 
[14] Kogeleschatz U. Dielectric-barrier discharge: their history, discharge physics,and industrial application. Plasma Chem and Plasma Proc 2003; 23 (1): 1-46.

[15] Zhang C, shao T, Yu Y, Niu Z and Yan P. Comparison of experiment and simulation on dielectric barrier discharge driven by $50 \mathrm{~Hz}$ AC power in atmospheric air. J. Electrostatics 2010; 68 (5): 445-52.

[16] Valdivia-Barrientos R, Pacheco-Sotelo V, Pacheco-Pacheco M, Benitez-Read J S and Lopez-Callejas R. Analysis and electrical modeling of a cylindrical DBD configuration at different operating frequencies. Plasma Sources Sci. Technol 2006; 15(2): 237-45.

[17] Pal UN, Sharma AK, Soni J, Khatun H and Kumar M. Electrical modeling approach for discharge analysis of a coaxial DBD tube filled with argon. J. Phys D:Appl. Phys 2009; 42(4).

[18] Raizer YP. Gas Discharge Physics. Berlin: Springer; 1991 chapter 3.

[19] Liu S and Neiger M. Electrical modeling of homogenous dielectric barrier discharge under an arbitrary excitation voltage. J Phys D: Appl Phys 2003; 36 (24) :3144-150.

[20] Jean-Louis Naudin, How to build yourself a Glow Discharge Plasma Panel, created on January 16th, 2000 - JLN Labs - Last update February 5, 2003, http://jnaudin.free.fr/html/s_gdp1.htm

[21] J. Shin and L. L.Raja .Dynamics of pulse phenomena in helium dielectric-barrier atmospheric-pressure glow discharges. J Phys D:Appl Phys 2003;94(12): 7408-415.

[22] Zhang YD, Gu B, Peng X and Wang DZ. Multiple current peaks and mode conversion of atmospheric pressure glow dielectric barrier discharge in helium. Thin Solid Films 2008; 516(21):7547-554. 